\title{
A budding surprise from the joint
}

\author{
Reactivation of dormant infections is increasingly recognised with \\ immunosuppression for rheumatic diseases. Septic arthritis from dimorphic fungi is \\ exceedingly rare in non-endemic settings. We describe the first Australian case of \\ Histoplasma capsulatum septic arthritis in a man of Laotian descent who was receiving \\ treatment for seropositive rheumatoid arthritis.
}

Andrew S Jordan BSc(Med) MB BS, FRACP Rheumatology Trainee

Ruchir Chavada MD,

Microbiology Trainee

Vanathi Nagendra MB BS,

Microbiology Trainee

H Patrick McNeil

MB BS, PhD, FRACP Professor of Rheumatology

Katherine Kociuba FRCPA, FRACP, Infectious Disease and Microbiology Physician²

Kathryn A Gibson

BM BCh, FRACP, PhD Director of Rheumatology

1 Liverpool Hospital, Sydney, NSW.

2 Sydney South West Pathology Service Sydney, NSW.

andrew@

bjchealth.com.au

doi: 10.5694/mjal3.10504

\section{Clinical record}

A 78-year-old man of Laotian descent but resident in Australia for many years was referred in October 2010 with bilateral wrist extensor tenosynovitis, and a diagnosis of seropositive rheumatoid arthritis was made (cyclic citrullinated peptide antibody level, $>200 \mathrm{U} / \mathrm{mL}$ [reference interval, <5 U/mL]; rheumatoid-factor negative). Although oral methotrexate initially improved disease control, he later had progressive disease involving his knees and ankles. Prednisone (5-15 mg daily) and leflunomide (10 mg daily) were added without much improvement, and he was considered for further immune modulation therapy. The patient's background included tuberculous cervical lymphadenitis in 2009 (positive result of culture for Mycobacterium tuberculosis), treated with 12 months of standard quadruple therapy.

In March 2012, the patient noticed worsening right ankle pain with synovitis, and a joint aspirate showed inflammatory synovial fluid (white cell count, $5100 \times 10^{6} / \mathrm{L}$ ) with negative results of bacterial cultures. Intra-articular corticosteroids provided symptomatic relief; however, the right ankle worsened in July 2012, and this time a repeat intra-articular corticosteroid injection failed to improve his symptoms. Due to ongoing synovitis in other joints and raised inflammatory markers, he was given a single $8 \mathrm{mg} / \mathrm{kg}$ dose of intravenous tocilizumab (a humanised, monoclonal, interleukin-6 receptor antibody). A repeat ankle aspiration and corticosteroid injection was performed concurrently. One week after the tocilizumab infusion, the right ankle synovitis became more prominent, and Histoplasma capsulatum was isolated from the most recent ankle aspirate. The diagnosis was confirmed by morphology of the yeast forms (Box, A), thermal dimorphism (mould phase converting to yeast form) (Box, B) and a panfungal polymerase chain reaction, which showed the same organism. He was systemically well with no fevers, sweats or weight loss. C-reactive protein was mildly raised at $10.4 \mathrm{mg} / \mathrm{L}$ (reference interval, $<5 \mathrm{mg} / \mathrm{L}$ ). Arthrotomy with debridement and lavage of the ankle joint was performed, and surgical specimens also showed $H$. capsulatum. Computed tomography scans of his brain, chest and abdomen did not show any evidence of disseminated disease. Further history showed he had frequently travelled to rural Laotian communities as a tourist before his diagnosis of

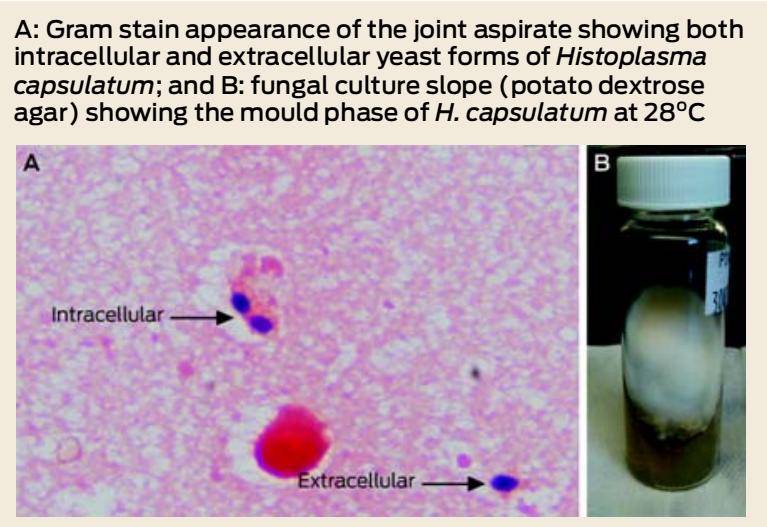

rheumatoid arthritis in 2010. After 2 weeks of induction therapy with intravenous liposomal amphotericin, treatment was maintained with oral itraconazole at therapeutic levels. Immunosuppression was minimised with cessation of methotrexate, leflunomide and tocilizumab, and gradual withdrawal of all corticosteroid treatment. He has made a steady recovery with no flare of his inflammatory arthritis to date.

\section{Discussion}

We believe this is the first report of $H$. capsulatum septic arthritis in Australia. H. capsulatum is a thermally dimorphic soil fungus, growing as a mould in the environment and as yeast at body temperature. ${ }^{1}$ It is endemic to North America, parts of Europe and South-East Asia (where our patient was born). Infection occurs with inhalation of spores aerosolised during activities that disturb soil. Initial infection is transient and asymptomatic in most of the immunocompetent population, with fewer than $5 \%$ of people estimated to be symptomatic. ${ }^{2}$ Macrophages play a key role in clearing histoplasma infection, and this is why disseminated disease occurs mostly in immunocompromised patients. Disseminated histoplasmosis has increasingly been reported in endemic areas after use of tumour necrosis factor-alpha (TNF- $\alpha$ ) inhibitors for rheumatoid arthritis. ${ }^{3}$ Histoplasmosis is three times more common than tuberculosis as a cause of serious infection in patients receiving TNF- $\alpha$ blockers. ${ }^{4}$ While a sterile immune-mediated 
polyarthritis is well recognised to occur in conjunction with symptomatic pulmonary histoplasmosis, ${ }^{5}$ localised septic arthritis is exceedingly rare, with only seven case reports in the literature. ${ }^{6}$

Tocilizumab has not been reported in association with histoplasmosis, and is unlikely to have been the cause in this case, as the diagnosis of septic arthritis was made at the same time as tocilizumab was administered. However, the additional immune suppression may have worsened the patient's symptoms.

A monoarthritis in a patient with a known diagnosis of an inflammatory polyarthritis such as rheumatoid arthritis is septic arthritis until proven otherwise. Atypical infectious organisms should be considered as a possibility in patients who are immunosuppressed, including those treated with monoclonal antibodies. Dormant infectious organisms can reactivate in the setting of immunosuppres- sion and can cause septic arthritis even in areas of low endemicity.

Competing interests: No relevant disclosures.

Received 20 Apr 2013, accepted 27 Jul 2013

1 Versalovic J, Carroll KC, Funke G, et al, editors. Manual of clinical microbiology. 10th ed. Washington DC: ASM Press, 2011.

2 Kauffman CA. Diagnosis of histoplasmosis in immunosupressed patients. Curr Opin Infect Dis 2008; 21: 421-425.

3 Lee JH, Slifman NR, Gershon SK, et al. Life-threatening histoplasmosis complicating immunotherapy with tumor necrosis factor alpha antagonists infliximab and etanercept. Arthritis Rheum 2002; 46: 2565-2570.

4 Hage CA, Bowyer S, Tarvin SE, et al. Recognition, diagnosis, and treatment of histoplasmosis complicating tumor necrosis factor blocker therapy. Clin Infect Dis 2010; 50: 85-92.

5 Rosenthal J, Brandt KD, Wheat LJ, Slama TG. Rheumatologic manifestations of histoplasmosis in recent Indianapolis epidemic. Arthritis Rheum 1983; 26: 1065-1070.

6 Fowler VG Jr, Nacinovich FM, Alspaugh JA, Corey GR. Prosthetic joint Infection due to Histoplasma capsulatum: case report and review. Clin Infect Dis 1998; 26: 1017. 